\title{
Evolution, dissolution and reversible generation of gold and silver nanoclusters in micelle by UV-activation
}

\author{
SUJIT KUMAR GHOSH, SUBRATA KUNDU and TARASANKAR PAL* \\ Department of Chemistry, Indian Institute of Technology, Kharagpur 721 302, India
}

\begin{abstract}
Gold and silver nanoparticles were produced separately by UV photoactivation (with variable flux density) in the presence of nonionic micelle, TX-100. Even their cyano complexes break down in TX-100 under UV and hence dissolution and reevolution of almost monodispersed nanoparticles $(\sim 3 \mathrm{~nm})$ are possible.
\end{abstract}

Keywords. Nanoparticle; micelle; photolysis; electrolytic effect.

\section{Introduction}

In this brief communication, we have highlighted for the first time the in situ preparation, dissolution and reversible generation of gold and silver nanoparticles from their cyano complexes by the UV-light. Here we have exhibited the preparation of gold and silver nanoparticles by UVphotoactivation method and then their dissolution in the presence of cyanide ion (cyano complexation of $\mathrm{Au}$ and $\mathrm{Ag}$ indeed!). The cyano complexes on further irradiation with UV-exposer regenerated the corresponding metal nanoparticles. The dissolution and regeneration steps were carried out reversibly up to a certain concentration of gold $\left(7 \times 10^{-5} \mathrm{M}\right)$ and silver $\left(3 \times 10^{-5} \mathrm{M}\right)$. For the evolution of gold, a catalytic amount of pre-formed $\mathrm{Au}(0)$ particle was needed but for silver there was no need of any trace element. A probable mechanism has been proposed for the reduction of $\mathrm{Au}(\mathrm{III})$ ion. The extent of formation of gold particles has also been investigated as a function of flux density (100-850 Lux) of the UV-light.

\section{Experimental}

Photochemical reaction was carried out in $1 \mathrm{~cm}$ quartz cuvette in a photoreactor with a variable flux density of 100-850 Lux. The flux density was measured using a Digital Lux Meter (model LX-101), Taiwan. UV-visible spectra were measured in Shimadzu UV-160 digital spectrophotometer (Kyoto, Japan) with $1 \mathrm{~cm}$ quartz cuvette. Transmission electron microscopy (TEM) studies of the particles were carried out at $200 \mathrm{kV}$ using a Hitachi HF-2000 TEM equipped with a field emission source. Aqueous $10^{-2} \mathrm{M} \mathrm{TX}-100,5 \times 10^{-3} \mathrm{M} \mathrm{HAuCl}{ }_{4}, 10^{-2} \mathrm{M}$ $\mathrm{AgNO}_{3}$ and $10^{-2} \mathrm{M}$ sodium cyanide $(\mathrm{NaCN})$ solutions were used as stock solution.

\footnotetext{
*Author for correspondence
}

\section{Results and discussion}

3.1 Preparation, dissolution and reversible generation of $A u(0)$ and $A g(0)$

Nanoparticle of $\mathrm{Au}(0)$ was obtained by the reduction of $\mathrm{HAuCl}_{4}$ in $\mathrm{TX}-100$ medium by $\mathrm{UV}$-activation following the reported method (Pal 1998) and the solution showed the plasmon absorption band having a $\lambda_{\max }$ at $523 \mathrm{~nm}$. The photo produced gold particles were dissolved using $\mathrm{NaCN}$ in the nonionic micelle in air (Jana et al 2000). The dissolution reaction is

$$
\begin{aligned}
& 4 \mathrm{Au}(0)+16 \mathrm{NaCN}+6 \mathrm{H}_{2} \mathrm{O}+3 \mathrm{O}_{2} \rightarrow 4 \mathrm{Na}\left[\mathrm{Au}(\mathrm{CN})_{4}\right] \\
& +12 \mathrm{NaOH}
\end{aligned}
$$

When the cyano complex of $\mathrm{Au}(\mathrm{III})$ was again photolysed in the presence of trace amount of gold, surprisingly same amount (same absorbance value at $523 \mathrm{~nm}$ ) of $\mathrm{Au}(0)$ was produced. The process can be carried out time and again for a gold concentration up to $7 \times 10^{-5} \mathrm{M}$ and cyanide concentration up to $3 \cdot 3 \times 10^{-5} \mathrm{M}$. Higher concentration of $\mathrm{HAuCl}_{4}$ or cyanide ion led to the aggregation of particles (electrolytic effect), which was concluded from red shifting of the peak position. Similar was the case of silver without the need of any trace metal.

The evolution, dissolution and reversible generation of $\mathrm{Au}$ and Ag nanoparticles in TX-100 micelle are depicted in scheme 1 .

Ultra fine size $(\sim 3 \mathrm{~nm})$ of gold and silver particles were produced which were authenticated from transmission electron microscopy (TEM) and are shown in figure 1 .

A probable mechanism has been proposed for the reduction of $\mathrm{Au}(\mathrm{III})$ ion (Gachard et al 1998; Fraccois et al 2000). The trivalent gold, Au(III) is reduced by the hydroxymethyl radical generated by the photolysis of TX-100 [R-0- $\mathrm{CH}_{2} \mathrm{CH}_{2} \mathrm{OH}$, where $\mathrm{R}=\left(\mathrm{CH}_{3}\right)_{3} \mathrm{CCH}_{2} \mathrm{C}\left(\mathrm{CH}_{3}\right)_{2}$ $\left.\mathrm{C}_{6} \mathrm{H}_{4}\left(\mathrm{OCH}_{2} \mathrm{CH}_{2}\right)_{\sim 9}-\right]$ into $\mathrm{Au}(\mathrm{I})$. The next step is the fast disproportionation of $\mathrm{Au}(\mathrm{I})$ and $\mathrm{Au}(\mathrm{III})$. Then, accumulated aurous ions $\mathrm{Au}(\mathrm{I})$ are reduced by the hydroxymethyl 


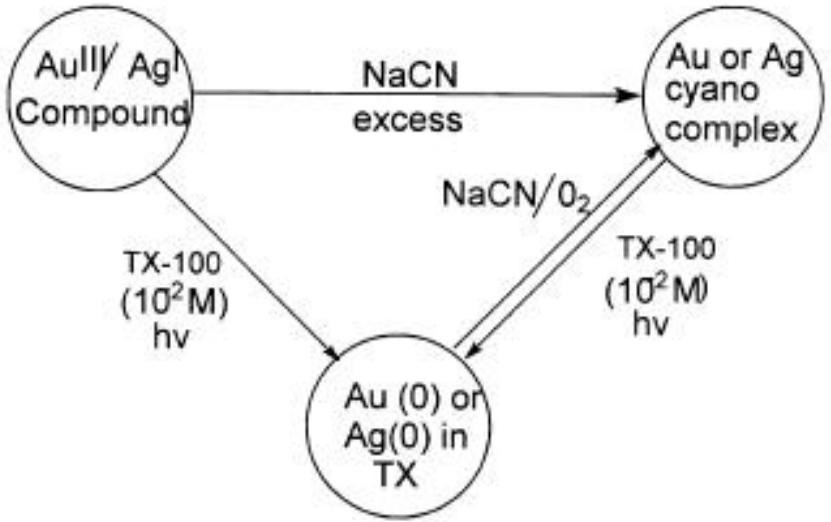

Scheme 1. Overall reaction steps showing the evolution, dissolution and reversible generation of $\mathrm{Au}$ or $\mathrm{Ag}$ nanoparticles.
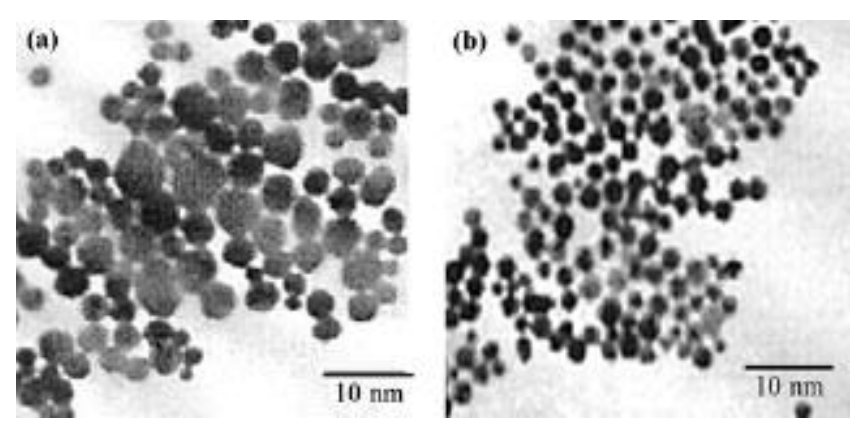

Figure 1. Transmission electron micrograph of (a) gold nanoparticles and (b) silver nanoparticles.

radical to $\mathrm{Au}(0)$. The atoms are formed with a homogeneous distribution throughout the solution. The atoms, then, tend to dimerise when encountered in pairs or associated with excess ions. But the micellar structures formed by the TX-100 molecules inhibit this association and thus act as particle stabilizer.

\subsection{Formation of $A u(0)$ as a function of flux density}

Lastly, the formation of $\mathrm{Au}(0)$ nanoparticles was investigated as a function of flux density (100-850 Lux) keeping the irradiation time constant $(12 \mathrm{~min})$. For a particular concentration of $\mathrm{TX}-100$ and $\mathrm{HAuCl}_{4}$, at a lower flux density ( 100 Lux $)$, the plasmon absorption band for

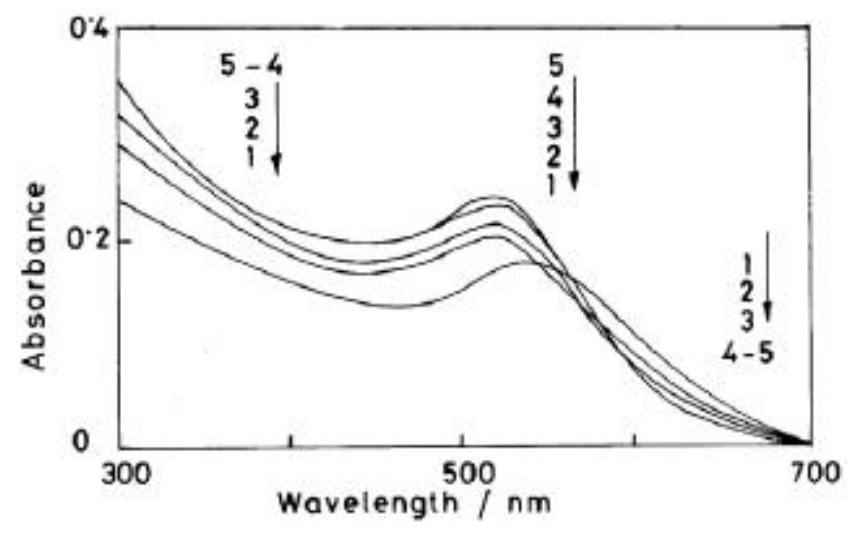

Figure 2. (1-5) Successive evolution of gold nanoparticles from $\mathrm{HAuCl}_{4}\left(7.4 \times 10^{-5} \mathrm{M}\right)$ in TX-100 medium $\left(0.99 \times 10^{-2} \mathrm{M}\right)$ with variable flux density (100-850 Lux) maintaining constant time of irradiation (12 min).

$\mathrm{Au}(0)$ appeared at $536 \mathrm{~nm}$. With the successive increase in flux density, the plasmon absorption band shifts progressively to the blue region with concomitant increase in absorbance value. Finally, at high density ( 850 Lux), the absorption band shifts to $519 \mathrm{~nm}$. The successive generation of gold nanoclusters is shown in figure 2 .

\section{Conclusion}

Nanoparticles of gold and silver can be generated reversibly from their cyano complexes. Smaller nanoparticles can be generated in comparison to the other methods. Variation in flux density brings about changes in the size of particles.

\section{Acknowledgement}

We gratefully acknowledge financial support from DST, New Delhi.

\section{References}

Fraccois L, Mostafavi M, Belloni J, Delouis J F, Delaire J and Feneyrou P 2000 J. Phys. Chem. B104 6133

Gachard E, Remita H, Khatouri J, Keita B, Nadjo L and Belloni J 1998 New J. Chem. 1257

Jana N R, Wang Z L and Pal T 2000 Langmuir 162457

Pal A 1998 Talanta 46583 